\title{
Silicone assisted, argon laser confinement of recurrent proliferative vitreoretinopathy related retinal detachment: a technique to allow silicone oil removal in problem eyes
}

\author{
D H W Steel, P Weir, C R H James
}

\begin{abstract}
Aims/background-Recurrent peripheral retinal detachments may occur in eyes treated with vitrectomy and silicone oil for retinal detachments complicated by proliferative vitreoretinopathy (PVR). The aim of this study was to assess whether laser photocoagulation could be used in the presence of silicone oil to confine and stabilise recurrent PVR related peripheral retinal detachments enabling the timely removal of the oil.

Methods-10 patients with recurrent peripheral retinal detachments after vitrectomy and silicone oil insertion were treated with posturing and subsequent focal argon laser to circumscribe the area of recurrent detachment.

Results-This technique alone was sufficient to limit the area of retinal detachment in seven of the cases. The remaining three cases required relieving retinotomies because of increasing retinal detachment despite the laser. In all 10 cases the silicone oil was later removed without progression of the detached areas.

Conclusion-Silicone assisted argon laser 'confinement' can be effective in stabilising eyes with peripheral retinal detachments allowing the subsequent removal of silicone oil.
\end{abstract}

(Br F Ophthalmol 1997;81:765-770)

Proliferative vitreoretinopathy (PVR) is the commonest cause of retinal detachment surgery failure. ${ }^{1}$ Despite vitrectomy and careful membrane dissection, recurrent traction in the vitreous base may lead to further peripheral tractional or combined tractional/ rhegmatogenous retinal detachment. ${ }^{12}$ Successful postoperative retinal tamponade has been achieved with both silicone oil and perfluoropropane gas. ${ }^{3}$ Silicone oil has the advantage of being a permanent tamponade agent and recently further analysis of the silicone study has shown its use to be associated with a better visual prognosis in those with anterior PVR. ${ }^{4}$ Unfortunately, silicone oil is not without significant ocular complications including cataract, ${ }^{5-7}$ glaucoma, ${ }^{58}$ perisilicone epiretinal membrane proliferation, ${ }^{9}$ emulsification, and keratopathy. ${ }^{5}$ These complications are partly related to the duration of intraocular exposure to silicone oil, ${ }^{5}{ }^{10}$ and once complications appear removal of the oil may not necessarily lead to their reversal. ${ }^{11}{ }^{12}$ Furthermore, visual function is often improved following silicone removal by eliminating the variability in refraction induced by the anterior curvature of the silicone bubble. ${ }^{11}$ Removing the oil, however, may result in macula redetachment, ${ }^{11}{ }^{13-15}$ especially in the presence of peripheral recurrent detachment before oil removal, requiring further surgery involving complex rebuckling procedures, repeated membrane dissection, and retinotomies. ${ }^{16}$ This is time consuming for both patient and surgeon as well as being related to a poorer visual outcome, ${ }^{1}$ and may result in the surgeon leaving the oil in the eye as a long term solution to stabilise the retina. Indeed, persisting retinal detachment was present in $59 \%$ of the eyes in the silicone study that did not undergo oil removal, and fear of recurrent macula detachment, and the resultant need for further surgery, appears to have been a common reason for not removing the silicone oil. ${ }^{11}$

Since the retinal redetachment rate does not appear to be influenced by the duration of intraocular oil, ${ }^{11}$ it seems reasonable to remove the oil as early as possible to avoid the initiation or worsening of oil associated complications.

The successful use of argon laser to 'delimit' selected non-PVR related rhegmatogenous retinal detachments has been described. ${ }^{17}$ The aim of this study was to assess whether argon laser photocoagulation used in conjunction with silicone oil could stabilise recurrent peripheral PVR related retinal detachments thus allowing oil removal before the onset of oil related complications.

\section{Method}

Ten consecutive patients who developed recurrent peripheral PVR related retinal detachments after vitrectomy and silicone oil exchange were entered into the study (see Fig 1).There were six male and four female patients with an age range of 37 to 87 years; five were phakic, four pseudophakic, and one aphakic. The degree of PVR ranged from C2 to D2 (Retina Society classification), ${ }^{18}$ and anterior PVR was present in five.

Seven patients were tertiary referrals from other centres after failed conventional retinal detachment surgery and persisting retinal detachment complicated by PVR. Two pa- 


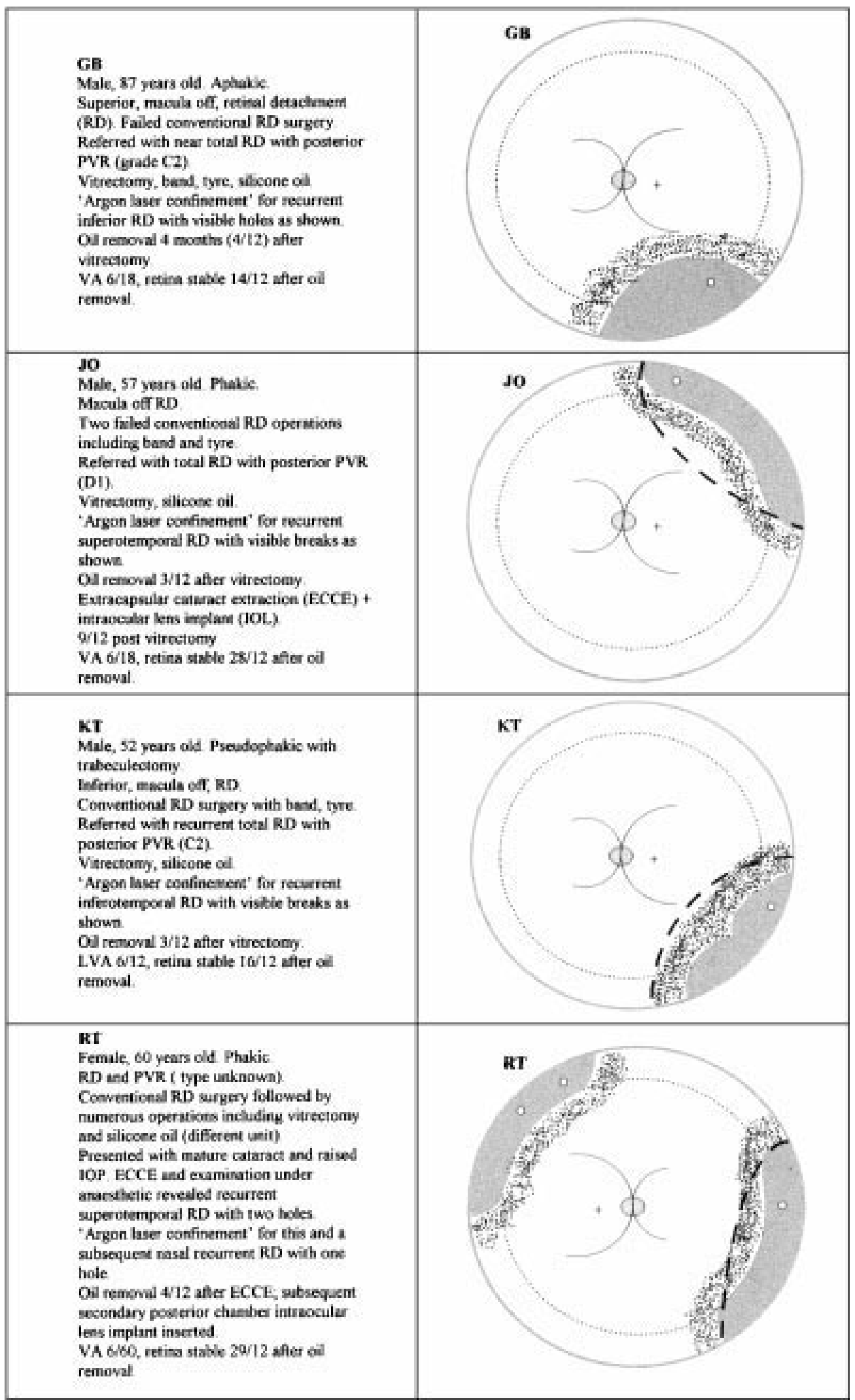

Figure 1 Details of 10 patients in study.

tients, who presented with established PVR without previous surgery, had a vitrectomy and silicone oil exchange as a primary procedure. Surgery consisted of a three port pars plana vitrectomy and membrane dissection with a careful basal vitreous trim and internal search. Anterior traction was released and subretinal fluid drained internally through existing retinal holes, if necessary with the use of heavy liquid to anteriorly displace posteriorly sequestered 


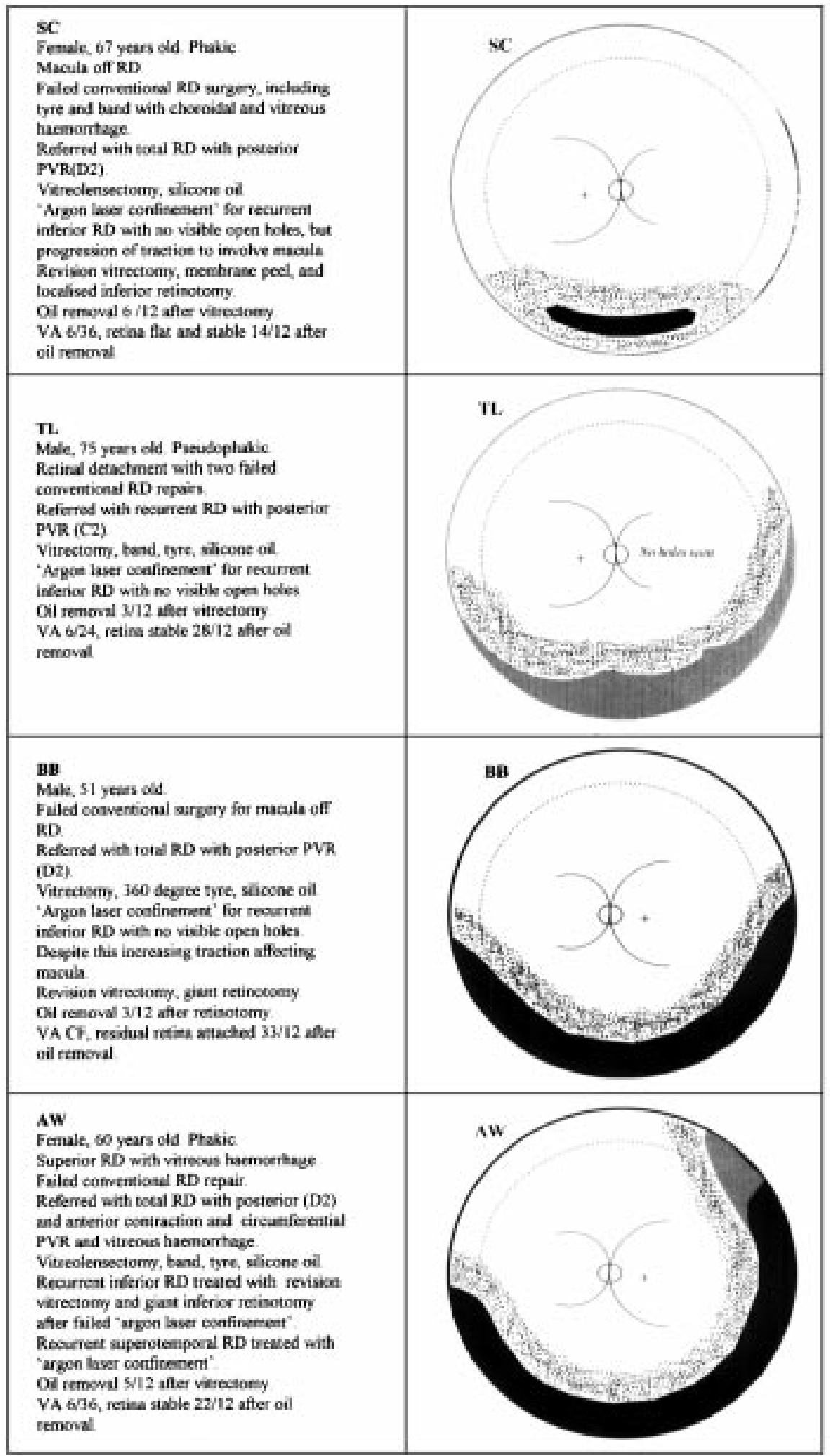

Fig 1 (cont)

fluid. Silicone oil exchange was followed by indirect argon laser to surround all visible retinal holes as well the peripheral retina posterior to the entry sites. An encircling scleral buckle was applied if not already present. Of the five phakic patients, two had a lensectomy performed at the time of the vitrectomy. All patients had a completely reattached retina at the completion of surgery. Face down posturing was performed with the cases as inpatients for an average of 6 days after surgery to establish firm chorioretinal adhesions at the site of laser. One patient (RT) presented from another unit with silicone oil in situ with raised intraocular pressure and a mature cataract. The exact details of the original surgery were 


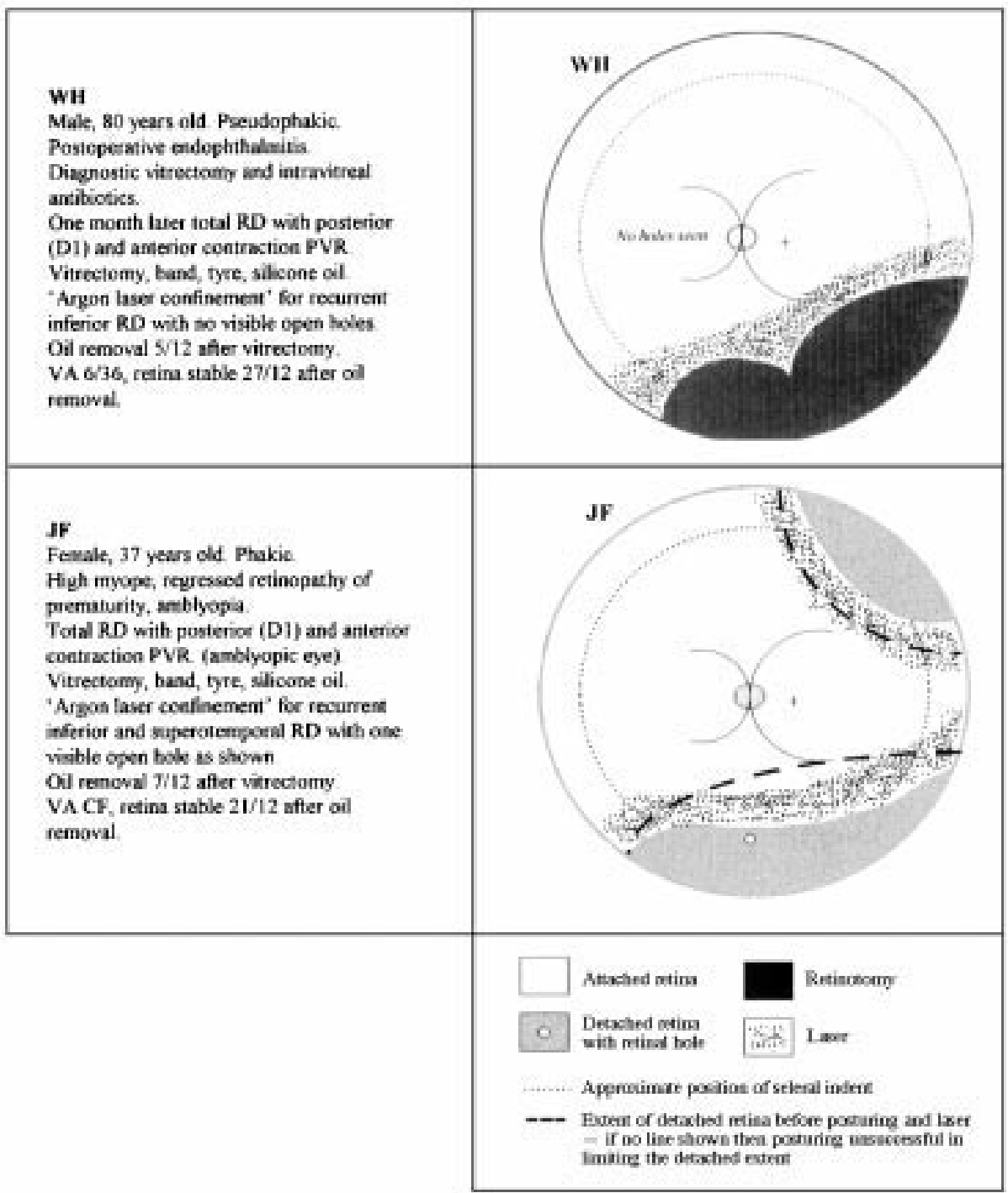

Fig 1 (cont)

unknown and recurrent peripheral retinal detachment was noted after cataract surgery.

Patients were carefully followed up after surgery for signs of recurrent retinal detachment. When this subsequently developed they were readmitted and postured in an attempt to limit the size of the retinal detachment and close any visible open retinal breaks. After this confinement of the retinal detachment, slit lamp delivered argon laser photocoagulation was applied to areas of flat retina surrounding the area of detachment using either a three mirror or Rodenstock contact lens.

A scatter pattern of three to four rows of moderate intensity white burns was used with a $200 \mu \mathrm{m}$ spot size and exposure time of $100 \mathrm{~ms}$. Care was taken to avoid high intensity burns which can lead to atrophic retinal holes in the presence of silicone. ${ }^{2}$ Areas of recurrent membrane formation were avoided to prevent aggravating traction already present.

Treatment was applied in two to three sessions and posturing was continued until a firm adhesion was established.

Patients were then observed for at least 2 months after the laser for signs of progression of the retinal detachment beyond the laser confines. If this did not occur and the retinal configuration remained stable then the silicone oil was removed. If progression of the detached retina did occur then further surgery was carried out before oil removal to stabilise the retina. Oil removal was accomplished through the pars plana in phakic and pseudophakic eyes and through the limbus in aphakic eyes. A balanced salt solution infusion was inserted and the silicone oil removed using active aspiration with a wide bore cannula.

\section{Results}

Silicone assisted argon laser confinement alone was successful in limiting and stabilising the recurrent peripheral detachments in seven out of the 10 eyes. In two of these eyes there were two separate areas of non-confluent peripheral detachment (JF, RT) and in five of the seven eyes the area of retinal detachment extended posterior to the encircling indent (WH, GB, KT, JF, TL) (Fig 1). Retinal holes associated with the recurrent detachments were identified in five of the seven eyes. These were thought to 


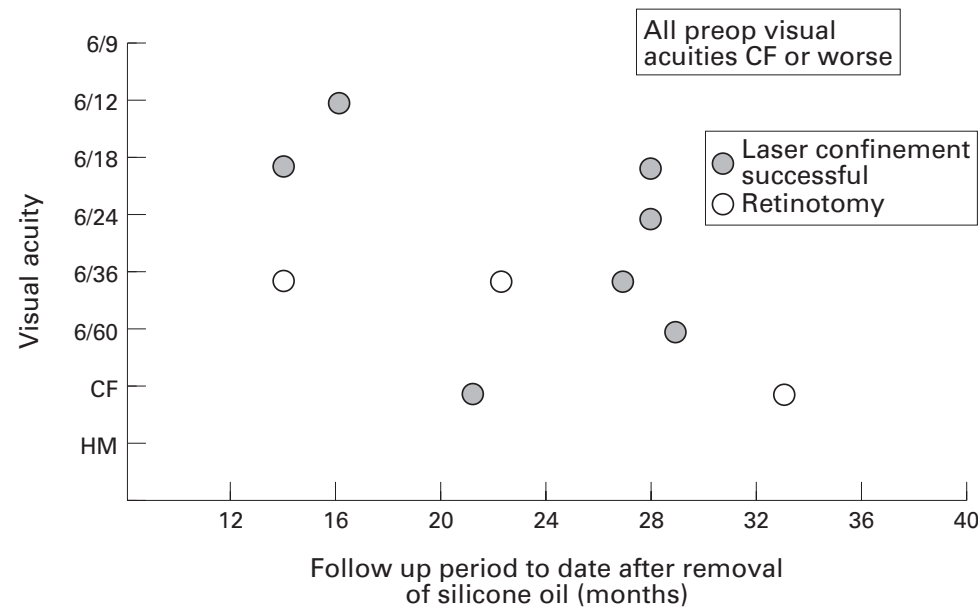

Figure 2 Graph of visual acuity versus length of follow up following silicone oil removal (CF = counting fingers vision, HM = hand movements vision $)$

be a reopening of original holes in three of the five cases, a new tractional hole in one case (GB), and in the other the details of the original surgery were unknown (RT). In two patients no open holes could be identified. Posturing was used successfully to reduce the extent of the retinal detachment in four of the seven cases (original extent of detached area shown as dotted lines on case diagrams, Fig 1). The retina in these cases has remained stable after oil removal with a mean follow up period of 23 months. Final visual acuities ranged from 6/12 to CF (Fig 2).

In three patients argon laser confinement was unsuccessful in stabilising the retina and retinotomies were performed before subsequent silicone oil removal. All three had rapidly progressive recurrent inferior detachments ultimately affecting the macula with very marked anterior PVR and no identifiable retinal holes. In one of these patients (AW) a subsequent area of recurrent detachment was treated successfully with laser confinement. Final visual acuities in these cases ranged from 6/36 to CF (Fig 2). All three of these cases had grade D2 posterior PVR as well as anterior PVR before vitrectomy.

COMPLICATIONS OF SILICONE OIL

Of the 10 patients, four were already pseudophakic, two had lensectomies at the time of vitrectomy, and one was already aphakic. Of the three remaining phakic patients, all three developed some degree of lens opacities, two subsequently requiring cataract surgery. One patient (SC) also developed a mild band keratopathy which was successfully removed, without subsequent recurrence. One patient (RT) had glaucoma at the time of presentation to this unit in association with prolonged insertion of silicone oil. Following silicone oil removal, intraocular pressure returned to the normal range on topical $\beta$ blockers only, although the optic disc was severely cupped. There were no cases of rubeosis or hypotony.

\section{Discussion}

The risk of recurrent retinal detachment involving the macula following silicone oil removal is well known. To decrease this risk, Gnad et al performed panretinal photocoagulation (PRP) in patients with reattached retinas before oil removal. ${ }^{19}$ Similarly, the rate of redetachment following silicone oil removal in diabetic eyes, in which PRP has previously been performed, is also reported to be low. ${ }^{20}$ In the presence of pre-existing peripheral retinal detachment before oil removal the risk of macula redetachment is even higher and the visual prognosis poor ${ }^{11}$ unless further measures are taken. While silicone oil remains in an eye it acts as a permanent tamponade agent and, even if retinal breaks reopen, the resultant detachment is limited as the new or reopened breaks come into contact and are closed by the silicone bubble. ${ }^{21}$ Ruellan and Roussat reported that PRP limited the posterior extension of recurrent peripheral retinal detachments anterior to an encircling indent following silicone oil removal. ${ }^{22}$ Lean has described the use of argon laser to circumscribe small superior recurrent retinal detachments in silicone filled eyes but noted that inferior recurrent detachments often needed repeat surgery with rebuckling or a retinotomy before oil removal. ${ }^{21}$ We have extended the use of this technique to include inferior retinal detachments, including ones extending posterior to encircling indents, by the use of posturing to reduce the size of the detachment and performing the argon laser while posturing is continued to create a firm chorioretinal scar confining the resulting limited area of detachment. PRP was not routinely performed and treatment was limited to the area surrounding the detached retina to avoid the possible increased risk of PVR associated with more extensive laser, and also to avoid unnecessary visual field loss.

Silicone assisted argon laser 'confinement' alone was effective in stabilising seven of the 10 eyes allowing the subsequent removal of the oil. In these seven patients further complex surgery was avoided, although we acknowledge that this may have been equally successful in retinal stabilisation. No eyes developed hypotony or rubeosis which have both been recorded with persistent peripheral retinal detachments. ${ }^{2324}$ Although this series is small the final visual acuity results in this group compare very favourably with other PVR series after silicone oil removal. ${ }^{1011} 14$ One of the seven patients (JF) was amblyopic in the affected eye and had a final acuity of counting fingers. One other patient (RT) had a final acuity of $6 / 60$ after prolonged installation of silicone oil before being seen in this unit, with raised intraocular pressure and severe optic disc cupping. The other five patients had acuities of $6 / 36$ or better. A poorer visual outcome has been noted in patients requiring complex surgery for recurrent PVR related retinal detachments and our good final acuity results may reflect this. However, we included patients in our study with lesser degrees of initial PVR and this may also explain the favourable final visual acuity results. All the patients successfully treated with laser confinement have now been followed up for at least 14 months after 


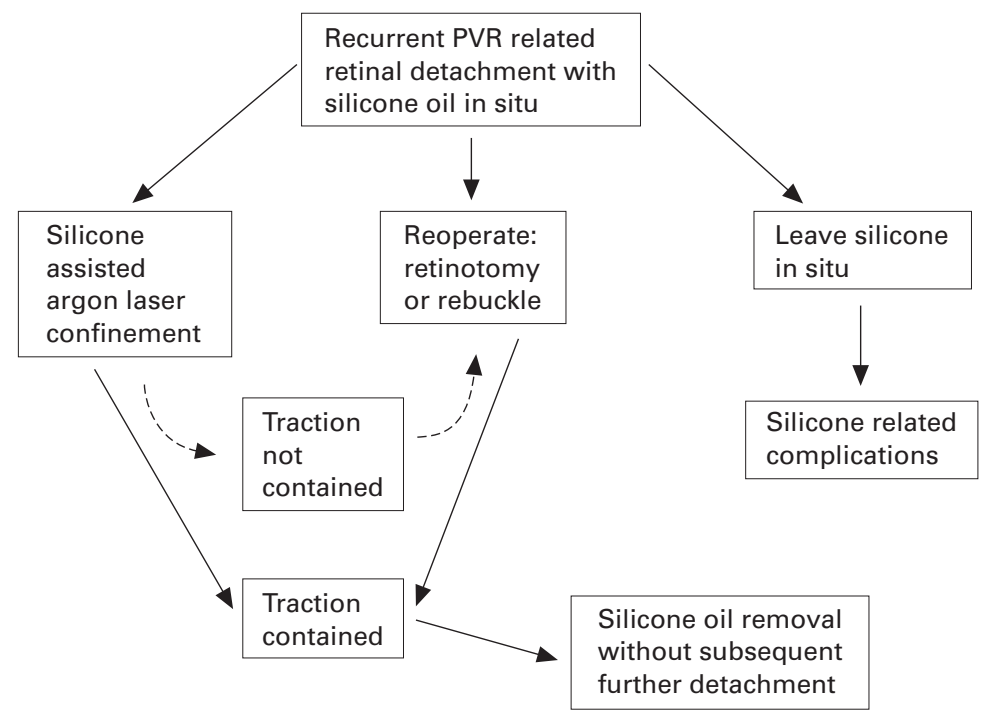

Figure 3 Flow diagram illustrating management of patients with recurrent PVR related peripheral retinal detachments with silicone oil in situ.

oil removal suggesting, therefore, that the retinal stabilisation achieved is likely to be long lived.

The technique was unsuccessful in three patients and retinotomies were needed to stabilise the retina before oil removal. The initial PVR in these three cases was more severe (grade D2 in addition to significant anterior PVR), and two of the cases had had preceding complicated conventional retinal detachment surgery with choroidal and vitreous haemorrhage. In all three cases the area of peripheral detachment and traction extended to involve the macula before oil removal despite posturing and laser demarcation. Very marked anterior PVR and surface epiretinal membranes were evident in the detached areas. Two of the cases involved more than two quadrants of peripheral retinal detachment. Although the numbers are small it is likely that argon laser confinement is less likely to be successful in these situations. In one of the three failed cases a further area of recurrent peripheral detachment after retinotomy was successfully treated with argon laser confinement and in all cases the silicone was later successfully removed with long term retinal stabilisation. The attempt at laser confinement did not adversely affect the final outcome.

Mietz and Heimann has shown that the median time interval between surgery and the onset or recurrence of PVR is 2 months. ${ }^{25}$ In this study the onset of the recurrent peripheral detachments occurred within $4-8$ weeks after surgery. The silicone oil was removed at a mean time of 4.3 months after vitrectomy when the retinal configuration was stable and at least 2 months after completion of the laser.

In this study silicone assisted argon laser confinement has proved to be a useful method of stabilising eyes with recurrent PVR related peripheral retinal detachment allowing early silicone oil removal and avoiding some of the potential complications of silicone. We believe consideration should be given to its use in this situation before more extensive operative procedures such as retinotomy or rebuckling (Fig 3).

1 Lewis $\mathrm{H}$, Aaberg TM. Anterior proliferative vitreoretinopathy. Am f Ophthalmol 1988;105:277-84.

2 Aaberg TM. XLV Edward Jackson Memorial Lecture. Management of anterior and posterior proliferative vitreoretinopathy. Am 7 Ophthalmol 1988;106:519-32.

3 The Silicone Study Group. Vitrectomy with silicone oil or perfluoropropane gas in eyes with severe proliferative vitreoperfluoropropane gas in eyes with severe proliferative vitreo-
retinopathy. Silicone Study Report 2. Arch Ophthalmol retinopathy. Silico

4 Diddie KR, Azen SP, Freeman MDC, Aaberg TM, Lewis $\mathrm{H}$, Radtke ND, et al. Anterior proliferative vitreoretinopathy in the silicone study. Silicone Study Report 10. Ophthalmology 1996;103:1092-9.

5 Casswell AG, Gregor ZJ. Silicone oil removal. I The effect on the complications of silicone oil. Br f Ophthalmol 1987; 71:893-7.

6 Baer RM, Aylward WG, Leaver PK. Cataract extraction following vitrectomy and silicone oil tamponade. Eye 1995;9: 309-12.

7 Leaver PK, Grey RHB, Garner A. Silicone oil injection in the treatment of massive pre-retinal retraction. II Late
complications in 93 eyes. Br $\mathcal{F}$ Ophthalmol 1979;63:361-7.

8 Barr CC, Lai M-Y, Lean JS, Linton KLP, Trese M, Abrams Barr CC, Lai M-Y, Lean JS, Linton KLP, Trese M, Abrams the silicone study. Silicone Study Report 4. Ophthalmology 1993;100:1629-35.

9 Lewis H, Burke JM, Abrams GW, Aaberg TM. Perisilicone proliferation after vitrectomy for proliferative vitreoretinopathy. Ophthalmology 1988;95:583-91.

10 Gonvers $M$. Temporary silicone oil tamponade in the management of retinal detachment in proliferative vitreoretinopathy. Am f Ophthalmol 1985;100:239-45.

11 Hutton WL, Azen SP, Blumenkranz MS, Lai M-Y, McCuen BW, Han DP, et al. The effects of silicone oil removal. SiliBW, Han DP, et al. The effects of silicone oil removal. Sili-
cone Study Report 6. Arch Ophthalmol 1994;112:778-85.

cone Study Report 6. Arch Ophthalmol 1994;112:778-85.
12 Moisseiev J, Barak A, Manaim T, Treister G. Removal of Moisseiev J, Barak A, Manaim T, Treister G. Removal of
silicone oil in the management of glaucoma in eyes with emulsified silicone. Retina 1993;13:290-5.

13 Franks WA, Leaver PK. Removal of silicone oil-rewards and penalties. Eye 1991;5:333-7.

14 Zilis JD, McCuen BW, de Juan Jr E, Steffansson E, Machemer R. Results of silicone oil removal in advanced proliferative vitreoretinopathy. Am f Ophthalmol 1989;108: 15-21.

15 Casswell AG, Gregor ZJ. Silicone oil removal. II Operative and postoperative complications. Br F Ophthalmol 1987;71: 898-902.

16 Hans DP, Lewis MT, Kuhn EM, Abrams GW, Mieler WF, Williams GA. Relaxing retinotomies and retinectomies. Williams GA. Relaxing retinotomies and retinectomies. Surgical results and predict.

17 Sigelman J. Laser therapy of peripheral retinal diseases. 1 Breaks and detachments. Ophthalmic Las Ther 1985;1:3549.

18 Retina Society Terminology Committee. The classification of retinal detachment with proliferative retinopathy. Ophthalmology 1983;90:121.

19 Gnad H, Skorpik C, Parousssis P, Radda TM, Klemmen UM, Lessel MR, et al. Funktionelle und anatomische resultate nach temporarer silikonolimplantation. Klin Monatsbl Augenheilkd 1984;185:364-7.

20 Pearson RV, McLeod D, Gregor ZJ. Removal of silicone oil following diabetic vitrectomy. Br f Ophthalmol 1993;77: 204-7.

21 Lean JS. Use of silicone oil as an additional technique in vitreoretinal surgery. In: Ryan S, ed. Retina. 2 nd ed. Vol 3. St Louis: CV Mosby, 1994; Chapter 133.

22 Ruellan YM, Roussat B. Decollement de retine: tamponnement interne provisoire par huille de silicone apres vitrectomie. F Fr Ophtalmol 1985;81:17-24.

23 van Meurs JC, Bolt BJP, Mertens DAE, Peperkamp E, de Waard P. Rubeosis of the iris in proliferative vitreoretinopathy. Retina 1996;16:292-5.

24 Lewis H, Verdaguer JI. Surgical treatment for chronic hypotony and anterior proliferative vitreoretinopathy. $\mathrm{Am} \mathcal{f}$ Ophthalmol 1996;122:228-35.

25 Mietz H, Heimann K. Onset and recurrence of proliferative vitreoretinopathy in various vitreoretinal diseases. $\mathrm{Br} F$ Ophthalmol 1995;79:874-7. 